\title{
A Unit Cost Adjusting Heuristic Algorithm for the Integrated Planning and Scheduling of a Two-Stage Supply Chain
}

\author{
Wang Jianhua, Huang Xianfeng, Mei Qiang, Tian Gang \\ Management School, Jiangsu University (China) \\ jzannywang@163.com,fengjianny@,163.com,qmei@ujs.edu.cn,jsyztg@126.com
}

Received: May 2014

Accepted: December 2014

\section{Abstract:}

Purpose: The stable relationship of one-supplier-one-customer is replaced by a dynamic relationship of multi-supplier-multi-customer in current market gradually, and efficient scheduling techniques are important tools of the dynamic supply chain relationship establishing process. This paper studies the optimization of the integrated planning and scheduling problem of a two-stage supply chain with multiple manufacturers and multiple retailers to obtain a minimum supply chain operating cost, whose manufacturers have different production capacities, holding and producing cost rates, transportation costs to retailers.

Design/methodology/approach: As a complex task allocation and scheduling problem, this paper sets up an INLP model for it and designs a Unit Cost Adjusting (UCA) heuristic algorithm that adjust the suppliers' supplying quantity according to their unit costs step by step to solve the model.

Findings: Relying on the contrasting analysis between the UCA and the Lingo solvers for optimizing many numerical experiments, results show that the INLP model and the UCA algorithm can obtain its near optimal solution of the two-stage supply chain's planning and scheduling problem within very short CPU time.

Research limitations/implications: The proposed UCA heuristic can easily help managers to optimizing the two-stage supply chain scheduling problems which doesn't include the delivery 
time and batch of orders. For two-stage supply chains are the most common form of actual commercial relationships, so to make some modification and study on the UCA heuristic should be able to optimize the integrated planning and scheduling problems of a supply chain with more reality constraints.

Originality/value: This research proposes an innovative UCA heuristic for optimizing the integrated planning and scheduling problem of two-stage supply chains with the constraints of suppliers' production capacity and the orders' delivering time, and has a great practical significance to the dynamic relationship establishment of multi-supplier-multi-customer in current market.

Keywords: supply chain, planning and scheduling, unit cost adjusting, UCA heuristic, dynamic relationship

\section{Introduction}

Due to the advanced information techniques, the competitions among corporations are replaced by these among supply chains gradually, and the stable cooperative relationships among corporations are evolved to dynamic cooperation with more flexibility and agility (Naim \& Gosling, 2011; Sharon \& DeGroote, 2013). In order to get competitive advantages among the global market, the companies of a supply chain need to take different policies to coordinate their purchasing, manufacturing and delivery processes so as to improve both the whole supply chain operating efficiency and each company's profit. By contrasting with supplier selection, partner relationship and coordination contracts, the task allocation planning and production scheduling also is a very important method to improve the supply chain dynamics and competitiveness.

Since Hall and Potts (2003) refers to the definition of Supply Chain Scheduling (SCS) as the study of coordination in scheduling decisions among the members of a supply chain, many scholars have studied the supply chain scheduling problem from different views. Some consider the supply chain scheduling and planning problems at the same time. Lasschuit and Thijssen (2004) explores the planning and scheduling of oil and chemical supply chains, gives some methods for making optimal decisions. Sawik (2009) proposes a mixed integer programming approach for solving a long-term, integrated scheduling of material manufacturing, material supply and product assembly in a customer driven supply chain. The overall problem is how to coordinate manufacturing and supply of parts and assembly of products such that the total supply chain inventory holding cost and the production line startup and parts shipping costs are minimized. Due to the complexity, Muñoz, Capón-García, 
Laínez-Aguirre, Espuña and Puigjaner (2015) decomposes the planning and scheduling problem to a master and a set of sub problems according to the information sharing and communicating processes in enterprises and builds some holistic mathematical models to help make decision.

Some study the online or dynamic scheduling problems of supply chains. Averbakh and Xue (2007) and Averbakh and Baysan (2012) study the two-level supply chain scheduling problems where customers release jobs to a manufacturer that has to process the jobs and deliver them to the customers under online and semi-online environment respectively, where offline means that both the resources and the orders information are available before scheduling, but online means that only the resources information is known. Ivanov and Sokolov (2012) develops an original approach to supply chain scheduling to answer the challenges of dynamics, uncertainty, and adaptivity by applying Pontryagin's maximum principle, and gives detailed description of the optimization of feedback loop-based dynamic and adaptive supply chain planning and scheduling .

Some research the transportation and inventory problems of supply chain scheduling. Agnetis, Hall and Pacciarelli (2006) gives a model of the scheduling coordination between a supplier and several manufacturers, shows that an intermediate storage buffer is benefit to resequence the jobs between the two stages. Chen and Pundoor (2006) considers how to find good processing and shipping schedules for a short selling season product supply chain with one manufacturer and many retailers. Chauhan, Gordon and Proth (2007) proposes a model and a real time algorithm for reducing supply chain's Work-In-Process (WIP) and improving the ability to respond to customer's requirements by coordinating the companies' production scheduling. Wu and Sarker (2013) develops an integrated inventory policy between a single producer and a multi-buyer in order to integrate the supply chain into a single actor in the face of competition to improve efficiency.

Some study the batch and delivery problems of supply chain scheduling. Selvarajah and Steiner (2006) studies the batch scheduling problem in a supply chain from the supplier's point of view where the supplier has to manufacture multiple products in large quantities and deliver them to customers in batches. The objective of the supplier is to minimize the inventory holding and delivery costs. Osman and Demirli (2012) presents a method for achieving the economic lot and delivery scheduling problem for a multi-stage supply chain comprising multiple items by developing a synchronized replenishment strategy, and specifying the sequence of production and the replenishment cycle time. Huang and Yao (2013) solves the optimal sequencing, lot-sizing and scheduling decisions for several products manufactured through several firms in a serial-type supply chain so as to minimize the sum of setup and inventory holding costs while meeting given demand from customers. Also, some literatures study the scheduling problems of the Make-to-Order supply chain or JIT supply chain in order to improve the agility and leanness of supply chain under uncertainty or customization 
marketplace (Demirli \& Yimer, 2008; Manoj, Gupta, Gupta \& Sriskandarajah, 2008; Yao, 2011; Subramanian, Rawlings, Maravelias, Flores-Cerrillo \& Megan, 2013).

This paper considers the integrated planning and scheduling optimization of a two-stage supply chain where the manufacturers' production capacities, production costs, holding costs and transportation costs are different, and the orders of retailers are known. In order to achieve the minimum operating cost of the supply chain, an INLP model is set up and a Unit Cost Adjusting (UCA) a heuristic algorithm is designed and verified.

The remainder of this paper is organized as follows. In Section 2, the problem definition with its mathematical formulation and possible applications are given. In Section 3, the proposed UCA heuristic is presented. In Section 4, some computational results and analysis are provided. Finally, some conclusions are summarized in Section 5.

\section{Problem Definition}

The first stage in the supply chain comprises $m$ suppliers with different production cost, stock cost rate and production capacity. In the second stage, we assume the existence of $n$ retailers whose demands known in advance need to be satisfied by a subset of the suppliers. The supply chain operates as a build-to-order style driven by the retailers' demands for only one kind of product.

For simplicity, we assume the finished goods transported from one supplier to one retailer by a single batch and the transportation times are negligible in comparison with production times. The transportation costs from each supplier to each retailer are different.

The objective is to minimize the total cost that includes the transportation cost, the production cost and the inventory cost of the supply chain. We aim to determine the supplying quantities from the suppliers to the retailers and the production scheduling of each supplier for its supplying product.

The notations and the mathematical model are given below:

\section{Parameters:}

$m=$ number of suppliers

$n=$ number of retailers

$i=$ supplier index $, I=1,2, \ldots, m$

$j=$ retailer index $, j=1,2, \ldots, n$ 
$Q_{j}=$ demand quantity of the $j$ th retailer

$C_{i}=$ production capacity of the $i$ th supplier

$c t_{i}=$ cycle time of the $i$ th supplier

$p c_{i}=$ unit production cost of the ith supplier

$h c_{i}=$ holding cost rate of the $i$ th supplier

$f c_{i, j}=$ fixed transportation cost from the $i$ th supplier to the $j$ th retailer

$v c_{i, j}=$ variable transportation cost from the $i$ th supplier to the $j$ th retailer

\section{Decision variables:}

$x_{i, j}=$ supplying quantity from the $i$ th supplier to the $j$ th retailer

\section{Mathematical formulation:}

(INLP)

$$
z_{P I N L P}=\min \sum_{i=1}^{m} \sum_{j=1}^{n}\left[h c_{i} \cdot x_{i j} \cdot c t_{i} \cdot \sum_{j^{\prime}=1}^{n} x_{i j^{\prime}}+p c_{i} \cdot x_{i j}+\left(f c_{i j}+v c_{i j} \cdot x_{i j}\right) \cdot \operatorname{sign}\left(x_{i j}\right)\right]
$$

Subject to:

$$
\begin{aligned}
& \sum_{i=1}^{m} x_{i j}=Q_{j} \quad \forall j \\
& \sum_{j=1}^{n} x_{i j} \leq C_{i} \quad \forall i \\
& x_{i j} \in \mathrm{N}+\quad \forall i, j
\end{aligned}
$$

The objective function (1) minimizes the total cost, constraint set (2) ensures that all of the suppliers can supply each retailer's demand, constraint set (3) indicates that the sum supplying quantity of each supplier is not bigger than its production capacity and constraint set (4) denotes the non-negative integer nature of the decision variables.

In the objective function formula (1), the production costs and the transportation costs can be understood easily, but the holding costs should be explained further. In any scheduling solution, if a supplier is chosen to produce some goods for retailers, the supplier is assumed to prepare all of the material before the production beginning, so the holding time length is the sum production time of the supplier's scheduled task. Therefore, the scheduling solution's holding cost is set as the first part of the formula (1) right expression. 
The proposed problem has many applications in manufacturing and service. For instance, a company with many retailers which designs and sales fashionable goods makes a new product promotion decision, so it needs choose the producers for all of its retailers' selling plans with different market capacities. Because the producers locate at different places and have different cost configuration and production capacities, the distances and transportation costs among the producers and the retailers are varied. The production task allocation and scheduling for its supply chain will affect the company's operation cost. Thus, the planning objective is to make the product supplying decision for each retailer such that the overall holding cost, production cost and distribution cost is minimal.

\section{The UCA Heuristic}

The INLP model can be obtained a local optimal solution by using LINGO software's nonlinear option with very long CPU time. However, the local solution is not the global one, and there also has some chance to improve the solution, meanwhile practical decisions could not wait for long calculating time. Therefore, it is necessary to develop an algorithm that can search an optimal solution for the INLP model within a reasonable CPU time in robustness. Since the objective of the INLP model is to find a minimum supply chain cost, we propose a heuristic algorithm that starts from any feasible initial solution, and finally get an optimal solution by adjusting the suppliers' supplying quantity according to their unit costs step by step, and name the algorithm as Unit Cost Adjusting (UCA) heuristic.

It is easy to understand a Multi-Retailer and multi-supplier Scheduling (MRS) problem can be divided into many Single-Retailer and multi-supplier Scheduling (SRS) problems. If all of the SRS problems are solved one by one, and the MRS problem will be solved. So the UCA heuristic includes three main procedures: (1) divide a MRS into some SRSs, and sort the SRSs so as to be solved one by one. (2) for each SRS, create an initial solution according to some conditions and then adjust the solution until an optimal solution obtained. (3) if all of the SRSs are solved, the MRS's optimal solution will be obtained.

\subsection{The UCA heuristic framework}

In this section, a framework of the UCA heuristic (Figure 1) which shows the optimization loop is presented and described in briefly, more details are presented in following sections.

Firstly, the algorithm need initialize the parameters for running, then sort the retailers by using BF or LF rules (Section 3.2), and finally do the main loop to achieve an optimal scheduling solution for each retailer in the sorted sequence one by one. 
In the main loop, for searching each retailer's optimal solution, an initial solution is created for the retailer based on its demand and the remaining capacity of suppliers by using different initialization strategies firstly (Details in section 3.3), and then the algorithm goes to two streams according to the condition of Failed Termination (FT) (Details in Section 3.4), if the FT is not met, the algorithm continues to loop, otherwise, the algorithm shuts down. When the FT is not met, the algorithm will calculates the unit cost of each supplier within the solution by using Formula (5), and then adjusts the quantities among the suppliers by using Formula (6) (Details in Section 3.5). After the adjustment, the solution is updated and the remaining capacities of the suppliers are updated too. Then the Successful Termination (ST) condition is judged whose result controls the algorithm to continue the retailer's schedule optimization or to do the next retailer's scheduling.

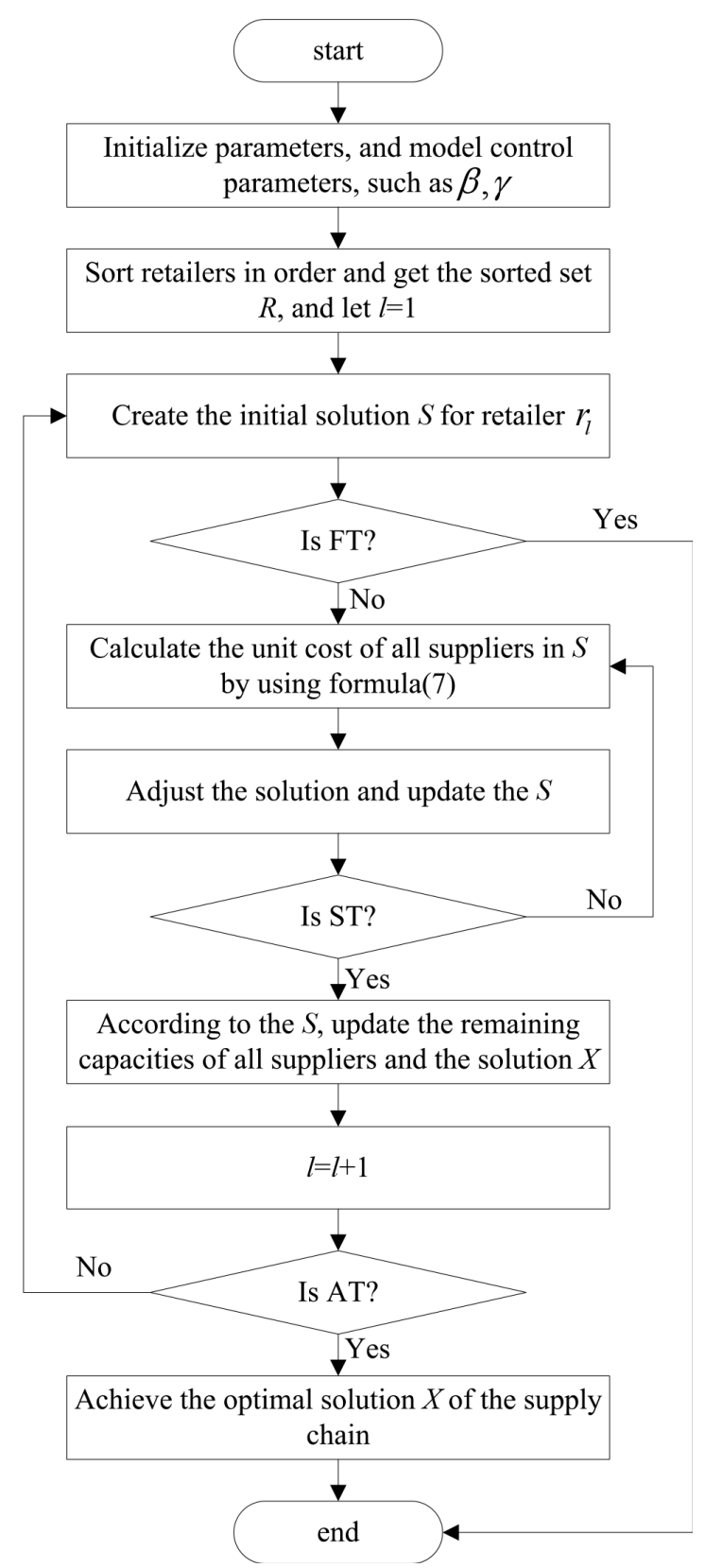

Figure 1. the UCA heuristic framework for MSP of a two-stage supply chain 


\subsection{Sorting rules for the SRSs}

After a MRS problem is divided into many SRS problems according to each retailer, the scheduling process needs a solving sequence of the SRS problems because the suppliers are common resources for the SRS problems. This paper proposes two common rules for scheduling problems' sorting which are named as Big First (BF) and little first (LF) respectively. A BF rule means the retailer whose demand is bigger than others will be met firstly, on the contrary, a LF rule means the retailer whose demand is fewer than others will be met firstly.

After the sorting process, a retailer index set $\mathrm{R}$ will be represented as following:

$$
R=\left\{r_{1}, r_{2}, \ldots, r_{l}, \ldots, r_{n}\right\}
$$

Where $Q_{r l} \leq Q_{r l^{\prime}}, \forall l>l^{\prime} \in[1, n]$ if adopting BF rule, $Q_{r l} \geq Q_{r l^{\prime}}, \forall l>l^{\prime} \in[1, n]$ if adopting LF rule.

\subsection{Initial solution strategies}

There have many strategies to create a feasible initial solution for a retailer's demand, such as All Demand for Fewest supplier (ADF), Mean Demand for Every supplier (MDE), Rate Demand for Every supplier (RDE). The ADF strategy tries to choose as few as possible suppliers to meet one retailer's demand. The ADF strategy means if there has a supplier whose capacity is not less than the demand, the supplier will be chosen to meet this retailer's demand; if all of the suppliers' capacities are less than the demand, the suppliers listed at front of the descending order list by their capacities will be chosen to meet the demand, so as to assure the quantity of suppliers chosen is the fewest. The MDE strategy spreads out the demand evenly to all of the suppliers without considering the suppliers' capacities. The RDE strategy assigns the demand to all of the suppliers by their capacities rate. The three strategies have their advantages respectively. The UCA algorithm will use all of the three strategies in turn to find a best solution within a solving process.

\subsection{Solution adjusting procedure based on unit costs}

In most condition, the initial solution is definitely not an optimal solution. The UCA heuristic need to adjust the initial solution according to the supplying unit cost of the suppliers involved in the solution until some termination conditions happen, and then the optimal solution is achieved. Because the objective of the scheduling optimization is to get a minimal cost, the intuition decision is to reduce the supplying quantity from the supplier whose unit cost is high, and increase the supplying quantity from the supplier whose unit cost is low. According to this 
principle, the adjusting process moves some product quantity from high cost suppliers to low cost suppliers.

Before to describe the adjusting procedures, the unit cost formula is given as (5).

$$
c_{i j^{*}}=\frac{h c_{i} \cdot x_{i j^{*}} \sum_{j=1}^{n} c t_{i} \cdot x_{i j}+p c_{i} \cdot x_{i j^{*}}+\left(f c_{i j^{*}}+v c_{i j^{*}} \cdot x_{i j^{*}}\right) \cdot \operatorname{sign}\left(x_{i, j^{*}}\right)}{x_{i j^{*}}} \quad \forall x_{i j^{*}}>0
$$

In formula (5), $j^{*}$ represents the scheduling retailer's index at present loop, and $c_{i j}$ is the unit cost of the $i$ th supplier for the $j$ th retailer in a solution which includes the three parts of the cost: holding cost, production cost and transportation cost. In the following section, if it doesn't create ambiguity, $c_{i}$ replaces $c_{i j}{ }^{*}$.

The adjusting process is described as following in detail:

Let set C (Figure 2) is the unit costs of suppliers of a solution in ascending order, where $n^{\prime}=n$, if the solution includes all of the suppliers; $n^{\prime}<n$, if the solution only includes part of the suppliers. The UCA heuristic's adjustment mechanism decides that the task assigned to a supplier with higher unit cost must be reduced, and the task assigned to one with lower unit cost must be increased. So, according to set $C$, the rear suppliers will give some supplying quantity to the front suppliers (Figure 2 ) in order, that is to say the $i_{n^{\prime}-k}$ supplier will give some supplying quantity to the $i_{k+1}$ supplier, where $0 \leq k \leq\left\lfloor n^{\prime} / 2\right\rfloor$.

$$
C=\left\{c_{i_{1}}, \overline{c_{i_{2}}}, \cdots, c_{i_{\mathrm{n}^{\prime}-1}}, c_{i_{\mathrm{n}^{\prime}}}\right\}
$$

Figure 2. unit cost set of a solution and the adjusting process diagram

Take supplier 1 and supplier 2 as example to explain the calculating rule for the Adjusted Quantity (AQ) between two suppliers, AQ is determined by formula (6).

$$
A Q=\min \left\{x_{2},\left[\frac{c_{2}}{c_{1}} \beta\right], C_{1}-x_{1}\right\}
$$

Where, $c_{1}, c_{2}$ represent the low unit cost and the high unit cost respectively, $x_{1}, x_{2}$ represent the supplying quantity of the suppliers with the low and high unit cost respectively; $\beta$ is the adjusting quantity base, $c_{1}$ represents the supplier 1 's capacity. Formulation (6) indicates that the bigger the gap between the high unit cost $c_{2}$ and the low unit cost $c_{1}$, the bigger the AQ value, and the $A Q$ value must be not bigger than the supplying quantity of the supplier 2 and the remaining supplying capacity of the supplier 1 . 


\subsection{Terminating conditions}

The UCA heuristic sets three terminating conditions for the scheduling optimization. For one retailer's supplying scheduling decision, the adjusting procedure will be continue forever if there is no terminating conditions, even if all of the suppliers involved in a solution whose unit costs are equal. So the first condition is named as Successful Termination (ST), whose basic rule is set as: if the adjusting procedure cannot get a better solution after $\gamma(\gamma=10)$ loops, this retailer's scheduling process is terminated. Obviously, while this condition appears, the retailer's scheduling is finished successfully.

Sometimes, the sum remaining capacity of all of the suppliers is less than the demand quantity of a retailer that is started its scheduling, so the UCA cannot create an initial solution for it. So the second condition is named as Failed Termination (FT), whose basic rule is set as: if an initial solution cannot be create, the supply chain scheduling process is terminated. Obviously, if this condition is activated, the scheduling process is fail.

Of course, if the second condition doesn't happen in a optimization process, the UCA heuristic will achieve an optimal solution for the supply chain scheduling problem after all of the retailers' demands are met in a better manner, and the algorithm is finished. So this condition is named as Algorithm Termination (AT).

\section{Computational Experiments}

\subsection{Experiments design}

Computational tests in this section are generated to verify and evaluate the performance of proposed UCA heuristic algorithm for solving the proposed PINIP model for the planning and scheduling problems of two stage supply chains. For this purpose, ten test problems with varying sizes of suppliers and retailers are generated at random in small and large-scale cases. Sizes of the test problems are given in Table 1. For each scale problem, 10 problem instances are randomly generated and the required parameters for these problems are extracted from uniform distributions whose ranges are listed in Table 2. The operation parameters are generated randomly by uniform distributions according to Table 2 . It is noteworthy that the

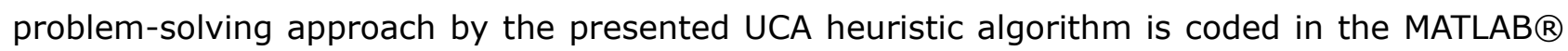
on a computer with the Intel Dual Core, $2.4 \mathrm{GHz}$ and $4 \mathrm{~GB}$ of RAM.

\begin{tabular}{|l|c|c|c|c|c|c|c|c|c|c|}
\hline Experiment No & 1 & 2 & 3 & 4 & 5 & 6 & 7 & 8 & 9 & 10 \\
\hline SupplierQty-m & 3 & 9 & 18 & 27 & 36 & 45 & 54 & 63 & 72 & 81 \\
\hline RetailerQty- $\boldsymbol{n}$ & 1 & 3 & 6 & 9 & 12 & 15 & 18 & 21 & 24 & 27 \\
\hline
\end{tabular}

Table 1. scales of each experiment 


\begin{tabular}{|l|c|c|c|c|c|c|c|}
\hline Parameters & $C_{i}$ & $Q_{j}$ & $c t_{i}$ & $h c_{i}$ & $p c_{i}$ & $f c_{i, j}$ & $v c_{i, j}$ \\
\hline Ranges & $(60,140)$ & $(100,200)$ & $(1,4)$ & $(0.01,0.06)$ & $(60,100)$ & $(100,200)$ & $(10,20)$ \\
\hline Unit & unit & unit & hour & \$/unit.hour & $\$ /$ unit & $\$$ & $\$ /$ unit \\
\hline
\end{tabular}

Table 2. the uniform distributions' ranges for the operation parameters

\subsection{Experimental results}

Table 3 gives the computational results of all the experiments, the mean costs and calculating times of each experiment's 10 instances solving by the UCA, and the ratios of the UCA/Lingo global solver and UCA/lingo nonlinear solver.

All of the 100 problem instances are solved by UCA within 2 seconds. In contrast, except for the instances of no 1 and no 2 experiments, we couldn't obtain the optimal solutions for the other test problems within a reasonable CPU time by using LINGO global solver. Meanwhile we could achieve local optimal solutions for all of the instances by running the LINGO nonlinear solver for a long CPU time.

\begin{tabular}{|c|c|c|c|c|c|c|}
\hline \multirow{2}{*}{ expNo } & \multicolumn{2}{|c|}{ UCA/LingoGlobal } & \multicolumn{2}{c|}{ UCA/Lingo Nonlinear } & \multicolumn{2}{c|}{ UCA results } \\
\cline { 2 - 7 } & time\% \% & cost $\%$ & time\% $\%$ & cost $\%$ & time (s) & cost \\
\hline 1 & 0.00 & 1.03 & 11.24 & 97.39 & 0.2 & 22715 \\
\hline 2 & 0.01 & 1.04 & 9.44 & 95.79 & 0.5 & 31868 \\
\hline 3 & - & - & 4.21 & 98.93 & 0.6 & 96065 \\
\hline 4 & - & - & 1.57 & 96.24 & 0.8 & 152031 \\
\hline 5 & - & - & 0.82 & 94.17 & 0.9 & 174071 \\
\hline 6 & - & - & 1.03 & 95.34 & 1.2 & 199830 \\
\hline 7 & - & - & 0.82 & 93.08 & 1.3 & 242962 \\
\hline 8 & - & - & 0.79 & 95.22 & 1.3 & 302623 \\
\hline 9 & - & - & 1.17 & 96.81 & 1.4 & 335534 \\
\hline 10 & - & - & 0.90 & 95.25 & 1.5 & 386542 \\
\hline
\end{tabular}

Note: - in the table represents that the Lingo global solver cannot obtain an optimal solution within 5 hours.

Table 3. computational results of the experiments

From Table 3, we can make a conclusion that the UCA algorithm can get a near optimal schedule for the SCS within a very short CPU time, so the UCA maybe a good algorithm for supply chain schedule optimization and can solve the planning and scheduling problems of two-stage supply chains effectively and efficiently. 


\section{Conclusions}

In this paper, we study the integrated planning and scheduling problem of a two-stage supply chain to minimize the sum cost of production and transportation for some certain orders. Since the problem is too complex to achieve an optimal solution within suitable calculating time by common algorithms, heuristic algorithms are good choices. In order to minimize the sum cost, we propose an UCA heuristic based on the idea that reducing the unit supplying costs of each product could lead to a minimal sum supplying cost of an order firstly. Then we verify that the UCA heuristic can solve the order allocation and production-scheduling problem simultaneously in large scale two-stage supply chain within a short CPU time by a lot of experiments. Because two-stage supply chains are the most common forms of actual commercial cooperation and any real complex supply chains are composed by two-stage supply chains, the proposed algorithm should have great effects on helping managers to build optimal supply chains and enlightening researchers to develop more practical algorithms for improving the supply chain's agility and flexibility.

The UCA heuristic can easily deal with the two-stage supply chain scheduling problem which doesn't include the delivery time and batch of orders, but in many real environments, the order delivery time and the delivery batch are very important factors of designing a supply chain network. Therefore, how to optimize the planning and scheduling problems of a supply chain with more reality constraints based on the UCA heuristic is a good opportunity for further research.

\section{Acknowledgements}

The study is supported by the National Natural Science Foundation of China (No. 71172191), the Ministry of Education, Humanities and Social Science Research Projects (No. 12YJA790123) the Excellent Talent Foundation of Jiangsu University (No. 11JDG006) and the post-doc fellowship of China for the first author.

\section{References}

Agnetis, A., Hall, N.G., \& Pacciarelli, D. (2006). Supply chain scheduling: Sequence coordination. Discrete Applied Mathematics, 154(15), 2044-2063.

http://dx.doi.org/10.1016/j.dam.2005.04.019

Averbakh, I., \& Baysan, M. (2012). Semi-online two-level supply chain scheduling problems. Journal of Scheduling, 15(3), 381-390. http://dx.doi.org/10.1007/s10951-011-0264-7 
Averbakh, I., \& Xue, Z.H. (2007). On-line supply chain scheduling problems with preemption. European Journal of Operational Research, 181(1), 500-504.

http://dx.doi.org/10.1016/j.ejor.2006.06.004

Chauhan, S.S., Gordon, V., \& Proth, J.M. (2007). Scheduling in supply chain environment. European Journal of Operational Research, 183(3), 961-970.

http://dx.doi.org/10.1016/j.ejor.2005.06.078

Chen, Z.L., \& Pundoor, G. (2006). Order assignment and scheduling in a supply chain. Operations Research, 54(3), 555-572. http://dx.doi.org/10.1287/opre.1060.0280

Demirli, K., \& Yimer, A.D. (2008). Fuzzy scheduling of a build-to-order supply chain. International Journal of Production Research, 46(14), 3931-3958.

http://dx.doi.org/10.1080/00207540601113273

Hall, N.G., \& Potts, C.N. (2003). Supply chain scheduling: Batching and delivery. Operations Research, 51(4), 566-584. http://dx.doi.org/10.1287/opre.51.4.566.16106

Huang, J.Y., \& Yao, M.J. (2013). On the optimal lot-sizing and scheduling problem in serial-type supply chain system using a time-varying lot-sizing policy. International Journal of Production Research, 51(3), 735-750. http://dx.doi.org/10.1080/00207543.2012.662604

Ivanov, D., \& Sokolov, B. (2012). Dynamic supply chain scheduling. Journal of Scheduling, 15(2), 201-216. http://dx.doi.org/10.1007/s10951-010-0189-6

Lasschuit, W., \& Thijssen, N. (2004). Supporting supply chain planning and scheduling decisions in the oil and chemical industry. Computers \& Chemical Engineering, 28(6-7), 863-870. http://dx.doi.org/10.1016/j.compchemeng.2003.09.026

Manoj, U.V., Gupta, J.N.D., Gupta, S.K., \& Sriskandarajah, C. (2008). Supply chain scheduling: Just-in-time environment. Annals of Operations Research, 161(1), 53-86.

http://dx.doi.org/10.1007/s10479-007-0290-1

Muñoz, E., Capón-García, E., Laínez-Aguirre, J.M., Espuña, A., \& Puigjaner, L. (2015). Supply chain planning and scheduling integration using Lagrangian decomposition in a knowledge management environment. Computers \& Chemical Engineering, 72(0), 52-67.

Naim, M.M., \& Gosling, J. (2011). On leanness, agility and leagile supply chains. Original Research Article. International Journal of Production Economics, 131(1), 342-354. http://dx.doi.org/10.1016/j.ijpe.2010.04.045

Osman, H., \& Demirli, K. (2012). Economic lot and delivery scheduling problem for multi-stage supply chains. International Journal of Production Economics, 136(2), 275-286. http://dx.doi.org/10.1016/j.ijpe.2011.12.001

Sawik, T. (2009). Coordinated supply chain scheduling. International Journal of Production Economics, 120(2), 437-451. http://dx.doi.org/10.1016/j.ijpe.2008.08.059 
Selvarajah, E., \& Steiner, G. (2006). Batch scheduling in a two-level supply chain - a focus on the supplier. European Journal of Operational Research, 173(1), 226-240.

http://dx.doi.org/10.1016/j.ejor.2004.12.007

Sharon, E., \& DeGroote, T.G.M. (2013). The impact of IT on supply chain agility and firm performance: An empirical investigation. International Journal of Information Management, 33(6), 909-916. http://dx.doi.org/10.1016/j.jijnfomgt.2013.09.001

Subramanian, K., Rawlings, J.B., Maravelias, C.T., Flores-Cerrillo, J., \& Megan, L.(2013). Integration of control theory and scheduling methods for supply chain management. Computers \& Chemical Engineering, 51, 4-20. http://dx.doi.org/10.1016/j.compchemeng.2012.06.012

Wu, B.Q., \& Sarker, B.R. (2013). Optimal manufacturing and delivery schedules in a supply chain system of deteriorating items. International Journal of Production Research, 51(3), 798-812. http://dx.doi.org/10.1080/00207543.2012.674650

Yao, J.M. (2011). Supply chain scheduling optimisation in mass customisation based on dynamic profit preference and application case study. Production Planning \& Control, 22(7), 690-707. http://dx.doi.org/10.1080/09537287.2010.537577

Journal of Industrial Engineering and Management, 2014 (www.jiem.org)

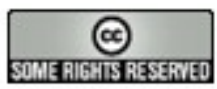

Article's contents are provided on a Attribution-Non Commercial 3.0 Creative commons license. Readers are allowed to copy, distribute and communicate article's contents, provided the author's and Journal of Industrial Engineering and Management's names are included. It must not be used for commercial purposes. To see the complete license contents, please visit http://creativecommons.org/licenses/by-nc/3.0/. 